\section{Theory of Mind Is Independent of Episodic Memory}

\author{
R. Shayna Rosenbaum, ${ }^{*}$ Donald T. Stuss, Brian Levine, Endel Tulving
}

A s humans, we are intrigued by who we are and how we differ from other creatures of evolution. Among the capacities thought to be uniquely human are autonoetic consciousness, the aspect of self-awareness that allows us to imagine our own experiences in different places at other times, and theory of mind (ToM), which allows us to infer other people's current mental states. The idea that ToM is closely related to, and that it may depend on, episodic memory and autonoetic consciousness seems perfectly natural: that in order to imagine and make sense of other people's thoughts, feelings, intentions, and actions, we must rely on our autobiographical recollections (1). The ability to consciously recollect past personal happenings has been shown to be necessary for imagining coherent and detailed personal happenings in the future $(2,3)$. Both episodic memory and ToM emerge close in time in ontogenetic development (4). The neural substrate on which the two abilities rely is in many ways strikingly similar (1).

In order to test the hypothesis that ToM requires autonoetic consciousness, it is necessary to administer objective ToM tests to participants without autonoetic abilities. Such tests are

Table 1. Performance of all participants on theory of mind (ToM) tests (8). Numbers in parentheses in left-hand column indicate maximum scores for each section.

\section{K. C. M. L. Controls [mean (SD)]}

First-order (/10)

False belief

Second-order (/10)

$10-10$

10

$10(0)$

Faux pas

$29 \quad 29$

$8.79(1.05)$

(/30)

Reading the mind in the eyes

26

31

$26.07(3.77)$

(/36)

Random appropriateness (/3)

Random intentionality (/5)

Goal-directed appropriateness (/3)

Goal-directed intentionality (/5)

ToM appropriateness (/3)

ToM intentionality (/5)

Sarcasm and empathy

First-order (/12)

Second-order (/12)

Animations

Visual perspective-taking/deception

Transfer of inference (/6)

Deception (first trial of five consecutive correct)

Emotional situations

Self (/8)

Other (/8)

$\begin{array}{ccc}3 & 3 & 2.64(0.46) \\ 0 & 0 & 0.48(0.58) \\ 3 & 2.75 & 2.36(0.41) \\ 2.75 & 2.5 & 2.64(0.29) \\ 3 & 2.5 & 2.2(0.5) \\ 4.5 & 4.5 & 3.66(0.7)\end{array}$

$12-11-11(124)$

$12 \quad 12$

$10.86(0.95)$

$5.93(0.27)$

$6.5(5.44)$

$7.64(0.5)$

$7.93(0.27)$ own past mental states is necessary to imagine the contents of other people's minds $(1,2)$. Both K. C. and M. L. suffer from severe difficulties in consciously (autonoetically) recollecting any events from any period of their lives. Yet they have no apparent difficulty in taking other persons' perspectives and inferring other people's thoughts, feelings, and intentions, as revealed by the ToM tests. The findings imply that K. C.'s and M. L.'s ToM ability may depend on semantic memory and general knowledge abilities that are largely preserved in both cases $(5,6)$.

Cases such as those of K. C. and M. L. allow for the study of ToM in isolation of autonoetic consciousness. Because these cases are rare, the relevant literature on the topic is sparse. The only other related piece of evidence of which we are aware is a report of a participant with a large medial prefrontal lesion who nonetheless performed well on several of the ToM tests used here. However, the extent to which his autobiographical memory impairment reflects a tendency to confabulate is unclear (7).

Our two cases do not shed any light on the extent to which fully functioning autonoetic ability is necessary for the development of ToM, because both K. C. and M. L. may have acquired ToM ability premorbidly. Our findings do, however, allow the conclusion that an existing severe impairment of episodic memory and autonoetic consciousness does not compromise the expression of ToM abilities. The dissociation we report here is important both theoretically and practically.

References and Notes

1. R. L. Buckner, D. C. Carroll, Trends Cogn. Sci. 11, 49 (2007).

2. D. Hassabis, E. A. Maguire, Trends Cogn. Sci. 11, 299 (2007).

3. D. L. Schacter, D. R. Addis, Nature 445, 27 (2007).

4. J. Perner, T. Ruffman, J. Exp. Child Psychol. 59, 516 (1995).

5. R. S. Rosenbaum et al., Neuropsychologia 43, 989 (2005).

6. B. Levine et al., Brain 121, 1951 (1998).

7. C. M. Bird, F. Castelli, O. Malik, U. Frith, M. Husain, Brain 127, 914 (2004).

8. Materials and methods are available on Science Online.

9. This research was supported by a Focus on Stroke Award from the Heart and Stroke Foundation (HSF) of Canada and the Canadian Institutes of Health Research and by grants from HSF of Ontario Centre for Stroke Recovery and Louis and Leah Posluns Centre for Stroke and Cognition at Baycrest. We thank R. Bloom, S. Gillingham, and L. Zackheim for technical assistance and F. Castelli and $\mathrm{U}$. Frith for making available the animations test.

\section{Supporting Online Material}

www.sciencemag.org/cgi/content/full/318/5854/1257/DC1 Materials and Methods

Fig. S1

Table S1

References

3 August 2007; accepted 10 October 2007

10.1126/science. 1148763

Department of Psychology, York University and Rotman Research Institute, Baycrest, Toronto, Ontario M3] 1P3, Canada.

*To whom correspondence should be addressed. E-mail: shaynar@yorku.ca 